\title{
Bactéries lactiques susceptibles d'inhiber la croissance de Clostridium tyrobutyricum en culture et dans le fromage
}

\author{
par \\ J. L. BERGERE, T. SYS et L. VASSAL \\ (avec la collaboration technique de MM. PITEL et CATHELIN \\ pour la fabrication des fromages) \\ Laboratoire de Biochimie Microbienne et Laiterie Expérimentale, \\ I.N.R.A. - 78350 Jouy-en-Josas (France)
}

Clostridium tyrobutyricum est le principal agent responsable du gonflement butyrique des fromages. L'apparition du défaut est avant tout fonction du nombre de spores de $C$. tyrobutyricum présentes dans le lait de fabrication. La contamination du lait a lieu à la production et dépend des soins apportés à la traite mais aussi et surtout du type d'aliment distribué aux vaches et de sa qualité bactériologique [9], la source principale de contamination étant presque toujours l'ensilage. Ensuite l'apparition du défaut est liée aux conditions qui règnent dans le fromage dans la mesure où elles peuvent contribuer à ralentir sinon einpêcher la croissance de C. tyrobutyricum, c'est le cas pour le $\mathrm{pH}$, la teneur en eau, la concentration en sel... Toutefois les fromages d'un type donné, ayant des caractéristiques identiques et fabriqués à partir de laits contenant les mêmes nombres de spores, ne présentent pas toujours les mêmes signes de gonflement butyrique. Ceci tend à prouver que d'autres facteurs jouent certainement un rôle dans l'apparition du défaut. On sait que des coliformes, des Bacillus mais aussi des bactéries lactiques de diverses espèces ainsi que des bactéries propioniques sont capables de stimuler la croissance et la production de gaz de $C$. tyrobutyricum [1, 14, 22, 32]. D'ailleurs cette bactérie ne fermentant que le lactate et un nombre limité de sucres [29], en particulier pas le lactose, il est nécessaire que ce sucre soit dégradé par d'autres bactéries pour qu'elle puisse l'utiliser. D'autre part puisqu'elle ne peut utiliser le lactate qu'en présence d'acétate et que ce dernier est de plus le principal promoteur de la germination de sa spore [2], il est évident que les bactéries produisant de l'acétate 
dans le fromage, y favorisent la croissance de C. tyrobutyricum. Inversement on sait que certaines souches de bactéries lactiques sont capables d'inhiber $C$. tyrobutyricum autrement que par l'acidification dont elles sont responsables. C'est le cas pour certaines souches de Streptococcus lactis productrices de nisine [13] et de lactobacilles, vraisemblablement grâce à l'eau oxygénée qu'ils produisent [6, 8]. D'autres souches de lactobacilles tels que $L$. casei, L. plantarum, $L$. acidophilus et $L$. helveticus ou même de streptocoques non producteurs de nisine sont également capables d'inhiber C. tyrobutyricum en culture sans qu'on ait identifié les substances qui en sont responsables $[7,12,20,24]$. Par conséquent on peut penser que les nombreux micro-organismes présents dans les fromages sont susceptibles de jouer un rôle dans l'apparition du gonflement butyrique soit parce qu'ils favorisent la croissance de C. tyrobutyricum, soit au contraire parce qu'ils la défavorisent.

A part l'utilisation de souches nisinogènes en fromagerie qui a fait l'objet de nombreux travaux [par ex. 8, 15, 23], celle d'autres souches inhibitrices a été relativement peu étudiée $[8,20]$. Or les phénomènes d'antagonisme pourraient être mis à profit pour lutter contre le gonflement butyrique.

C'est dans ce but que nous avons entrepris le présent travail. Nous avons d'abord recherché des souches inhibitrices de $C$. tyrobutyricum parmi des bactéries lactiques et surtout celles qui sont utilisées ou se développent naturellement dans les fromages. Ces bactéries ont été ensuite utilisées dans la fabrication de fromages d'Emmental afin de voir si leur activité inhibitrice est suffisante pour y empêcher le développement de $C$. tyrobutyricum et donc s'il serait possible de les employer pour contrôler le gonflement butyrique. D'autre part bien que cela n'ait pas été le but de l'étude nous avons tenté de déterminer la nature des facteurs inhibiteurs en cause.

\section{MATERIEL ET METHODES}

\section{Souches}

Les souches dont on a examiné les aptitudes inhibitrices se répartissaient de la manière suivante : 35 Lactobacillus bulgaricus, 32 L. lactis, 31 L. casei, 26 L. helveticus, $18 \mathrm{~L}$. plantarum, 8 L. acidophilus, 5 L. jugurti, 4 L. salivarius, 3 L. fermenti, 2 L. buchneri, $2 \mathrm{~L}$. brevis, $1 \mathrm{~L}$. leichmanii, $1 \mathrm{~L}$. cellobiosus et $1 \mathrm{~L}$. species ; 35 Streptococcus thermophilus, $17 \mathrm{~S}$. diacetylactis, $9 \mathrm{~S}$. cremoris, $8 \mathrm{~S}$. lactis, 3 S. durans, 2 S. faecalis, 2 S. species, 2 Leuconostoc citrovorum, 1. L. dextranicum, 3 pédiocoques. Ces souches provenaient soit de notre collection, soit d'autres collections internationales et leurs 
origines étaient variées. Etant donné leur nombre, seule l'origine des souches intéressantes sera précisée dans les résultats.

Les souches de $C$. tyrobutyricum étaient CNRZ 500, 510, 514, 518, 595, 596 et 608 ; elles provenaient de notre collection [29] et ont été isolées à partir de laits, de fromages et d'ensilages.

Les autres souches utilisées étaient Propionibacterium arabinosum P9 et $P$. freundenreichii subsp. shermanii CNRZ 435.

\section{Sélection des souches inhibitrices}

L'activité inhibitrice des souches de bactéries lactiques était déterminée par la méthode de diffusion en gélose. Le milieu RCM gélosé était inoculé avec une suspension de spores de $C$. tyrobutyricum CNRZ 510 activées par la chaleur [2] à raison de $10^{\circ}$ spores par $\mathrm{ml}$ et réparti en boîtes de Petri. On déposait à la surface du milieu six à huit disques de papier stériles (diam. $9 \mathrm{~mm}$ pour tests antibiotiques) qui étaient ensuite imprégnés d'une goutte de la culture à examiner. Ces dernières étaient effectuées sur lait tournesolé incubé à $30^{\circ} \mathrm{C}$ ou $37^{\circ} \mathrm{C}$ suivant qu'il s'agissait de mésophiles ou de thermophiles et prélevées peu de temps après le caillage du lait. Dans le cas de souches se développant mal sur le lait, on ajoutait à celui-ci 10 p. 100 de milieu MRS. Les boîtes de Petri étaient ensuite incubées pendant 2 à 3 j dans les jarres anaérobies en présence de Gas Pak. L'effet inhibiteur était alors mesuré par la zone d'inhibition de la croissance de $C$. tyrobutyricum. Tous les essais ont été effectués en double.

D'autre part les souches inhibitrices ont été essayées sur les sept souches de $C$. tyrobutyricum ensemencées sous forme de spores ou de cellules végétatives dans le milieu RCM gélosé, du MRS gélosé et du milieu au lactate gélosé dans les mêmes conditions que précédemment. Enfin de la même manière on a examiné leur influence sur les bactéries propioniques et les souches de bactéries lactiques utilisées comme levains dans la fabrication des fromages.

\section{Nature des substances inhibitrices}

La présence d' $\mathrm{H}_{2} \mathrm{O}_{2}$ dans les cultures sur lait des souches inhibitrices a été recherchée par iodométrie. La production $\mathrm{d}^{\prime} \mathrm{H}_{2} \mathrm{O}_{2}$ au cours du test d'inhibition en gélose a été recherchée par la technique au dioxyde de manganèse et celle à la benzidine [36]. Enfin on a étudié l'effet de la catalase sur l'inhibition en ajoutant une solution de cette enzyme au milieu gélosé ensemencé juste avant répartition en boîte de manière à obtenir une concentration finale de $10 \mu \mathrm{g} / \mathrm{ml}$ [6]. On vérifiait que la catalase était encore active après les 2 à $3 \mathrm{j}$ d'incubation. 
Dans le but de localiser les substances inhibitrices, des cultures ont été effectuées en milieu MRS puis centrifugées à 5000 g, les culots ainsi que les surnageants obtenus concentrés ou non, tels quels ou chauffés à $100^{\circ} \mathrm{C}$ pendant $5 \mathrm{mn}$, ont été testés sur le milieu gélosé. Les culots de cellules remis en suspension dans du tampon phosphate $0,03 \mathrm{M}$ à $\mathrm{pH} 7,0$ ont également été soumis à l'action des ultrasons (Siduse US 77-7 à $23 \mathrm{khz}$ ) pendant $20 \mathrm{mn}$, centrifugés et le culot ainsi que le surnageant obtenus testés pour l'activité inhibitrice. On a procédé de même avec des cultures autolysées en eau au lieu d'être désintégrées. On a effectué aussi des cultures sur lait ou en milieu MRS pendant les périodes plus ou moins longues, de 1 à $5 \mathrm{j}$, dans un appareil à dialyse et testé périodiquement le dialysat et la culture.

Enfin des cultures des souches inhibitrices ont été réalisées sur milieu MRS et RCM gélosé à 0,7 p. 100 . Le milieu était réparti en erlenmeyer de $50 \mathrm{ml}$ en couche de $1 \mathrm{~cm}$ environ d'épaisseur, stérilisé, inoculé en surface avec environ $1 \mathrm{ml}$ de préculture en milieu liquide puis incubé en jarre anaérobie pendant $48 \mathrm{~h}$ à $72 \mathrm{~h}$ à $30^{\circ} \mathrm{C}$ ou $37^{\circ} \mathrm{C}$. La gélose était centrifugée et le liquide surnageant était testé pour son activité inhibitrice après filtration sur membrane (Millipore - 0,45 $\mu \mathrm{m}$ ).

\section{Fabrications fromagères}

Des fromages d'Emmental ont été fabriqués selon le procédé habituel en utilisant un levain lactique normal constitué d'une souche de $L$. helveticus, CNRZ 303 et d'une souche de S. thermophilus, CNRZ 302 ainsi qu'un ensemencement du lait en bactéries propioniques, $P$. arabinosum P9 ou $P$. freudenreichii subsp. shermanii CNRZ 435.

A part le cas des fromages de contrôle, le lait de fabrication était également ensemencé avec une suspension de spores de $C$. tyrobutyricum (mélange de cinq souches) à raison de 2-5 spores par $\mathrm{ml}$. En outre des cultures actives des différentes souches inhibitrices préparées sur lait autoclavé étaient ajoutées, seules ou en mélange, au lait de fabrication un peu avant l'emprésurage à raison de 0,1 p. 100 au total, ceci quel que soit le nombre de souches sauf pour L. helveticus 45 qui était toujours ajouté à 0,1 p. 100 , ou 0,2 p. 100 lorsqu'il était le seul lactobacille thermophile (deuxième série). Trois séries de fabrications ont été ainsi réalisées à plusieurs mois d'intervalle. Les différentes combinaisons de souches utilisées figurent dans les résultats. Pour chaque série, deux fois par semaine, deux fromages étaient fabriqués à partir du même lait ensemencé avec deux souches inhibitrices différentes ou le mélange sauf dans la première série qui constituait un essai préliminaire. La succession des souches et de leur mélange était organisée suivant un plan en blocs incomplets équilibrés de façon à éliminer l'influence des conditions jour- 
nalières de fabrication. Dans la deuxième série, pour les lactobacilles se développant lentement sur le lait, les précultures ont été effectuées dans du lait additionné d'extrait de levure, $1 \mathrm{~g}$ par litre ; de $\mathrm{Mg}$ $\mathrm{SO}_{4} 7 \mathrm{H}_{2} \mathrm{O}, 0,2 \mathrm{~g}$ par litre et de $\mathrm{MnSO}_{4} 4 \mathrm{H}_{2} \mathrm{O}, 0,05 \mathrm{~g}$ par litre, ce milieu permettant une croissance plus rapide.

Pour chaque fromage on mesurait le $\mathrm{pH}$ au centre et en talon après $6 \mathrm{~h}$ de pressage et le lendemain, soit $24 \mathrm{~h}$ après le début de la fabrication, de manière à apprécier l'allure de son acidification. A $24 \mathrm{~h}$ on mesurait également son extrait sec. On notait le temps de séjour en cave chaude ce qui donnait une indication sur la vitesse d'ouverture des fromages et l'apparition du gonflement butyrique.

En fin d'affinage les fromages étaient coupés, leur ouverture notée et on effectuait un examen organoleptique. La présence d'un gonflement butyrique était confirmée (ou non) par un dénombrement de $C$. tyrobutyricum. En outre sur une partie des fromages on a recherché la présence d'inhibiteurs de $C$. tyrobutyricum et dénombré les lactobacilles mésophiles. Pour le pouvoir inhibiteur on a utilisé la même technique que celle qui a servi à sélectionner les souches. Le disque de papier était remplacé par un disque de fromage (diam. 8-10 mm, épaisseur 1-2 $\mathrm{mm}$ ) déposé dans une cupule de même diamètre découpée dans le milieu gélosé ensemencé en spores de $C$. tyrobutyricum. Les lactobacilles mésophiles étaient dénombrés en milieu MRS gélosé dans des tubes profonds incubés à $20^{\circ} \mathrm{C}$, avec confirmation par observation microscopique de quelques colonies. Ces examens étaient pratiqués sur les fromages le lendemain de leur fabrication et en fin d'affinage.

\section{RESULTATS}

\section{Sélection des souches inhibitrices}

La technique de diffusion en gélose a été choisie parce que c'est celle qui convient le mieux pour l'examen d'un grand nombre de souches d'autant plus qu'elle permet d'éviter les inhibitions par simple acidification que l'on peut observer par la technique en milieu liquide. Parmi les 252 souches examinées, une vingtaine exerçaient un effet stimulant sur la croissance de $C$. tyrobutyricum CNRZ 510 (colonies plus denses autour du disque), seulement neuf exerçaient une inhibition et le reste était sans effet. Trois $L$. helveticus ne provoquant qu'une zone d'inhibition très étroite et de manière non reproductible, six souches inhibitrices seulement ont été retenues (tab. 1). Ces dernières inhibaient également les autres souches de $C$. tyrobutyricum testées, les zones étant plus ou moins étroites. A part une exception elles n'avaient aucun effet sur les souches de 
bactéries utilisées couramment comme levains en fromagerie et dans ces essais : S. lactis C10, S. cremoris C7, S. diacetylactis DRCl, L. helveticus 303, S. thermophilus CNRZ 302, P. arabinosum P9 et P. freudenreichii subsp. shermanii CNRZ 435. La seule exception concerne les deux souches de $L$. plantarum qui inhibaient légèrement $S$. lactis et un peu plus L. helveticus, les largeurs des zones d'inhibition étant respectivement de moins de 0,5 $\mathrm{mm}$ et d'environ $1 \mathrm{~mm}$.

\section{TABLEAU 1}

Souches inhibitrices de $C$. tyrobutyricum CNRZ 510

\begin{tabular}{l|c}
\hline \multicolumn{1}{c|}{ Souche } & $\begin{array}{c}\text { Largeur des zones d'inhibition* } \\
(\mathrm{en} \mathrm{mm})\end{array}$ \\
\hline L. plantarum 3 & 3 \\
L. plantarum CNRZ 343 & 3 \\
L. casei K6 & 4 \\
L. casei CNRZ 316 & 3,5 \\
L. helveticus 45 & 2,5 \\
S. diacetylactis CNRZ 190 & 3 \\
& \\
\hline
\end{tabular}

* Ceci représente la largeur de l'anneau dépourvu de colonies qui entourait le disque de papier et non le diamètre de la zone d'inhibition ; milieu RCM.

\section{Nature de l'inhibition}

L'inhibition se produisait aussi bien lorsque le milieu était ensemencé avec des cellules végétatives de $C$. tyrobutyricum qu'avec des spores. Dans le cas des spores l'examen microscopique des prélèvements effectués dans la zone d'inhibition montrait qu'elles avaient perdu leur réfringence mais ne dépassaient pas ce stade ce qui signifiait que ce n'est pas la phase initiale mais la phase de croissance de la germination qui était inhibée.

L'inhibition n'était pas due à l'acide lactique apporté par la culture ou formé dans les disques par les cellules au cours de l'incubation. La présence d'indicateur coloré (pourpre de bromocrésol) dans le milieu n'a pas montré de zone acide autour du disque et l'addition de tampon phosphate au milieu ne supprimait par l'inhibition. D'ailleurs celle-ci se produisait aussi bien dans le 
milieu au lactate que dans un milieu (RCM ou MRS) contenant un sucre fermentescible par les souches inhibitrices. Enfin seulement six souches sur deux-cent-cinquante-deux s'étaient révélées inhibitrices alors que toutes produisaient de l'acide lactique et certaines même très activement.

Certaines souches de bactéries lactiques inhibant $C$. tyrobutyricum ou d'autres bactéries $[3,6,10,16,35]$ grâce à la production $\mathrm{d}^{\prime} \mathrm{H}_{2} \mathrm{O}_{2}$, nous avons examiné cette éventualité. Les précultures sur le lait ne contenaient pas ce composé en quantité dosable par iodométrie. Il ne s'en formait pas non plus dans les zones d'inhibition entourant les disques. Enfin l'addition de catalase dans le milieu de culture ne supprimait pas l'inhibition provoquée par les six souches alors qu'elle annulait l'effet inhibiteur d'un disque imprégné d' $\mathrm{H}_{2} \mathrm{O}_{2}$.

Dans le but de déterminer la nature de l'inhibition, des cultures des deux souches les plus actives, L. casei K6 et L. plantarum 343, ont été effectuées en milieu MRS liquide. Elles présentaient la même activité que les cultures sur le lait mais étaient plus faciles à traiter. Après centrifugation on a constaté que l'activité était liée aux cellules, le surnageant même concentré dix fois n'ayant aucun effet sur $C$. tyrobutyricum (tab. 2) de même d'ailleurs que les

\section{TABLEAU 2}

Influence de divers traitements sur l'inhibition de C. tyrobutyricum par L. casei $\mathrm{K} 6$ et $L$. plantarum 343

\begin{tabular}{|c|c|c|}
\hline \multicolumn{2}{|c|}{ Traitement ou fraction } & Inhibition \\
\hline \multicolumn{2}{|c|}{ Dialysat de culture } & - \\
\hline \multicolumn{2}{|c|}{ Centrifugation : - surnageant } & - \\
\hline & - culot & + \\
\hline Chauffage : & $100^{\circ} \mathrm{C} 5 \mathrm{mn}$ & - \\
\hline \multicolumn{2}{|l|}{ Chloroforme } & - \\
\hline \multirow[t]{2}{*}{ Ultrasons : } & — fraction soluble & - \\
\hline & - culot* & + \\
\hline \multirow[t]{2}{*}{ Lyse en eau : } & - fraction soluble & - \\
\hline & - culot & - \\
\hline
\end{tabular}

* Contenait encore des cellules viables. 
dialysats de cultures de différents âges. Le chauffage des cellules à $100^{\circ} \mathrm{C}$ ou leur traitement par le chloroforme supprimait l'inhibition. Lorsque les cellules étaient désintégrées par les ultrasons l'activité inhibitrice ne se retrouvait pas dans la partie soluble mais seulement dans les débris cellulaires. Toutefois dans cette dernière fraction il restait des cellules viables, de l'ordre de 1 à 10 p. 100 suivant les essais, dénombrables sur milieu de culture. Par contre le culot et le surnageant de centrifugation de suspensions de cellules incubées en eau pendant $48 \mathrm{~h}$, en grande partie lysées et ne contenant plus de cellules viables, ne présentaient aucune activité.

Lorsqu'on intercalait un morceau de membrane de dialyse entre les disques imprégnés des cultures des six souches et le milieu ensemencé en $C$. tyrobutyricum on n'observait aucune inhibition.

Des fragments de milieu gélosé prélevés autour des disques dans les zones d'inhibition ont été déposés sur du milieu RCM ensemencé en $C$. tyrobutyricum. Après incubation, pour cinq des souches inhibitrices étudiées, on observait des zones d'inhibition d'un diamètre de $12-15 \mathrm{~mm}$ mais on constatait qu'il y avait eu croissance des bactéries lactiques au niveau du fragment de gélose. La zone d'inhibition autour des disques étant étroite, il était en effet difficile de prélever du milieu qui n'ait pas été en contact avec le disque et donc dépourvu de cellules de bactéries lactiques. Cependant dans le cas d'une souche au moins, il n'y avait ni colonies, ni croissance décelable à l'examen microscopique dans le fragment de gélose et cependant on observait une plage d'inhibition d'un diamètre de 5 à $6 \mathrm{~mm}$. Celle-ci n'était donc pas due à la présence de cellules de la souche de lactobacilles inhibiteurs mais bien à une substance présente dans la gélose et formée au cours de l'incubation précédente. La production de composés inhibiteurs en milieu gélosé et non en milieu liquide a déjà été observée chez d'autres bactéries [37]. Afin de le vérifier, des cultures des six souches inhibitrices ont été effectuées sur milieu RCM et MRS gélosés à 0,7 p. 100 [37]. Après incubation, les extraits de culture débarrassés de cellules n'exerçaient pas d'effet inhibiteur sur $C$. tyrobutyricum lorsqu'ils étaient essayés par la méthode de diffusion.

\section{Utilisation des souches inhibitrices dans des fabrications d'Emmental}

Un premier essai préliminaire a d'abord été réalisé et ne comportait qu'un petit nombre de fromages fabriqués avec addition des souches inhibitrices (tab. 3). Sauf ceux fabriqués avec $S$. diacetylactis aucun ne présentait de défaut butyrique même localisé bien que dans le cas de $L$. casei l'un des fromages contenait un nombre de $C$. tyrobutyricum suffisant pour qu'on puisse conclure à un développement de cette bactérie. Après pressage les caractéristiques 


\section{TABLEAU 3}

Influence des souches inhibitrices sur l'apparition du gonflement butyrique dans des fromages d'Emmental (essai préliminaire)

\begin{tabular}{|c|c|c|c|c|c|c|}
\hline \multirow{3}{*}{$\begin{array}{l}\text { Souches inhibitrices } \\
\text { ajoutées (1) }\end{array}$} & \multicolumn{4}{|c|}{$\mathrm{pH}$ des fromages à } & \multirow{3}{*}{$\begin{array}{l}\text { Extrait } \\
\text { sec } \\
\text { en p. } 100 \\
\text { à } 24 \mathrm{~h}\end{array}$} & \multirow{3}{*}{$\begin{array}{l}\text { Nombre de } \\
\text { fromages } \\
\text { butyriques } \\
\text { (2) }\end{array}$} \\
\hline & \multicolumn{2}{|c|}{$6 \mathrm{~h}$} & \multicolumn{2}{|c|}{$24 \mathrm{~h}$} & & \\
\hline & C & $\mathrm{T}$ & $\mathrm{C}$ & $T$ & & \\
\hline \multicolumn{7}{|l|}{ Témoin sans } \\
\hline C. tyrobutyricum & 5,52 & 5,45 & 5,12 & 5,12 & 61,2 & \\
\hline L. plantarum $3+343$ & 5,30 & 5,16 & 5,07 & 5,14 & 60,4 & \\
\hline L. casei $\mathrm{K} 6+316$ & 5,5 & 5,40 & 5,14 & 5,12 & 61,0 & $1 \mathrm{Ba}$ \\
\hline L. helveticus 45 & 5,24 & 5,08 & 5,19 & 5,17 & 60,6 & \\
\hline S. diacetylactis 190 & 5,36 & 5,24 & 5,13 & 5,11 & 60,8 & $1 \mathrm{~B}$ \\
\hline Mélange six souches & 5,10 & 5,15 & 5,07 & 5,15 & 60,0 & \\
\hline
\end{tabular}

(1) Deux fromages fabriqués par espèce : l'un avec C. tyrobutyricum, l'autre sans, et quatre pour le mélange : deux avec et deux sans C. tyrobutyricum. Les résultats indiqués représentent les moyennes, $\mathrm{C}=$ Centre et $\mathrm{T}=$ Talon.

(2) $\mathrm{B}$ = fromage présentant un gonflement butyrique d'après l'aspect de l'ouverture et l'analyse.

$\mathrm{Ba}=$ ouverture non typiquement butyrique mais développement de $C$. tyrobutyricum dans le fromage d'après l'analyse.

$(\mathrm{pH}$, extrait sec) des différents fromages étaient à peu près les mêmes mais les valeurs du $\mathrm{pH}$ à $6 \mathrm{~h}$ montraient que l'acidification était le plus souvent plus rapide que dans les témoins ne comportant que les levains traditionnels et ceci surtout dans le cas du mélange de souches. D'une manière générale les fromages étaient d'autant moins ouverts que l'acidification était plus rapide. Tous les fromages fabriqués exerçaient une inhibition sur la croissance de $C$. tyrobutyricum par la méthode de diffusion en gélose, que ce soit $24 \mathrm{~h}$ après la fabrication, au début du passage en cave chaude ou à la fin de l'affinage. Les deux fromages témoins étaient inhibiteurs en fin d'affinage mais pas à $24 \mathrm{~h}$. A partir de l'un d'eux on a d'ailleurs 
isolé une souche de lactobacille inhibitrice appartenant vraisemblablement à l'espèce $L$. casei.

Une deuxième série de fromages a été fabriquée pour confirmer ces résultats mais pour chaque espèce inhibitrice on a utilisé une seule souche et on n'a pas testé le mélange complet des différentes espèces. En outre on a abandonné $S$. diacetylactis. Les fromages d'essai présentaient des caractéristiques à $24 \mathrm{~h}$ semblables à celles des témoins (tab. 4). Toutefois, comme dans l'essai préliminaire, l'acidification était plus rapide lorsque les souches inhibitrices, sauf L. plantarum, étaient présentes. Les fromages ouvraient à peu près en même temps sauf ceux fabriqués avec $L$. plantarum qui ouvraient aussi vite que les témoins butyriques sans souche inhibitrice (tab. 4). D'ailleurs deux d'entre eux présentaient un gonflement butyrique apparent ainsi que trois de ceux fabriqués avec la souche inhibitrice de $L$. helveticus seule c'est-à-dire sans autre lactobacille thermophile, chez ces derniers le gonflement était cependant plus tardif. Les fromages fabriqués normalement avec addition de $L$. casei et $L$. helveticus étaient exempts de défauts butyriques bien que deux d'entre eux contenaient un nombre important de $C$. tyrobutyricum. Tous les fromages y compris les témoins exerçaient un effet inhibiteur plus ou moins marqué sur la croissance de $C$. tyrobutyricum lorsqu'ils étaient examinés par la méthode de diffusion en gélose. Les dénombrements effectués pour vérifier si les lactobacilles mésophiles inhibiteurs se développaient dans les fromages n'ont pas été concluants. En effet tous les fromages contenaient dès le $2^{\text {e }}$ jour ce type de lactobacilles en nombre variant de $10^{4}$ à $10^{8}$ par $\mathrm{g}$; seuls, ceux avec addition de $L$. plantarum présentaient en général des nombres plus élevés, en moyenne $7.10^{7}\left(10^{6}\right.$ à $\left.2.10^{8}\right)$ alors que dans les autres les nombres n'excédaient pas $10^{7}$. La souche inhibitrice de $L$. helveticus se développait bien puisque lorsqu'elle était utilisée seule avec le levain de $S$. thermophilus, l'évolution du $\mathrm{pH}$ dans les fromages était correcte et même plus rapide que dans les témoins (tab. 4).

Enfin une troisième série de fromages a été fabriquée avec les mêmes combinaisons de souches inhibitrices que dans la première à l'exception de $S$. diacetylactis. Mais les résultats étaient nettement moins bons, en effet dans chaque lot, une partie des fromages présentaient des défauts butyriques apparents, et même tous dans le cas de $L$. casei (tab. 5). Cependant les défauts apparaissaient un peu plus tardivement que dans les fromages sans souches inhibitrices.

Malgré ces irrégularités, si on considère globalement les trois séries d'essais, la proportion de fromages ensemencés avec des souches inhibitrices et présentant un défaut butyrique apparent était de 43 p. 100 ( 57 p. 100 si on compte également ceux contenant un nombre élevé de $C$. tyrobutyricum) au lieu de 90 p. 100 pour les fromages fabriqués sans souche inhibitrice. 


\section{TABLEAU 4}

Influence des souches inhibitrices sur l'apparition du gonflement butyrique dans les fromages d'Emmental (deuxième essai)

\begin{tabular}{|c|c|c|c|c|c|c|c|}
\hline \multirow{3}{*}{$\begin{array}{l}\text { Souches inhibitrices } \\
\text { et } C \text {. tyrobutyricum }(\mathrm{Ct}) \\
\text { ajoutés (1) }\end{array}$} & \multicolumn{4}{|c|}{$\mathrm{pH}$ des fromages à } & \multirow{3}{*}{$\begin{array}{l}\text { Extrait sec } \\
\text { en p. } 100 \\
\text { à } 24 \mathrm{~h}\end{array}$} & \multirow{3}{*}{$\begin{array}{l}\text { Temps de } \\
\text { séjour en } \\
\text { cave chaude }\end{array}$} & \multirow{3}{*}{$\begin{array}{l}\text { Nombre de } \\
\text { fromages } \\
\text { butyriques (2) }\end{array}$} \\
\hline & \multicolumn{2}{|c|}{$6 \mathrm{~h}$} & \multicolumn{2}{|c|}{$24 \mathrm{~h}$} & & & \\
\hline & $\mathrm{C}$ & $\mathrm{T}$ & $\mathrm{C}$ & $\mathrm{T}$ & & & \\
\hline- & 5,75 & 5,58 & 5,16 & 5,17 & 62,1 & 59,4 & \\
\hline- & 5,69 & 5,54 & 5,12 & 5,16 & 61,8 & 47,2 & $4 \mathrm{~B}$ \\
\hline L. plantarum $3+\mathrm{Ct}$ & 5,72 & 5,50 & 5,17 & 5,15 & 62,1 & 45,0 & $2 \mathrm{~B}$ \\
\hline L. casei $\mathrm{K} 6+\mathrm{Ct}$ & 5,51 & 5,30 & 5,12 & 5,10 & 61,3 & 68,6 & $1 \mathrm{Ba}$ \\
\hline L. helveticus $45+\mathrm{Ct}$ & 5,46 & 5,32 & 5,11 & 5,11 & 61,4 & 61,6 & $1 \mathrm{Ba}$ \\
\hline L. helveticus $45 \mathrm{~S}+\mathrm{Ct}$ & 5,51 & 5,38 & 5,12 & 5,08 & 61,4 & 59,0 & $3 \mathrm{~B}+1 \mathrm{Ba}$ \\
\hline
\end{tabular}

(1) Pour chaque type cinq fromages ont été fabriqués; dans la fabrication $\mathrm{S}$ avec L. helveticus 45 , seule cette souche était utilisée comme lactobacille thermophile en plus de la souche de Streptococcus thermophilus habituelle.

(2) Voir légende du tableau 3. 
TABLEAU 5

Influence des soúches inhibitrices sur l'apparition du gonflement butyrique dans les fromages d'Emmental (troisième essai)

\begin{tabular}{|c|c|c|c|c|c|c|c|}
\hline \multirow{3}{*}{$\begin{array}{l}\text { Souches inhibitrices } \\
\text { et } C \text {. tyrobutyricum }(\mathrm{Ct}) \\
\text { ajoutés (1) }\end{array}$} & \multicolumn{4}{|c|}{$\mathrm{pH}$ des fromages à } & \multirow{3}{*}{$\begin{array}{l}\text { Extrait } \\
\text { sec } \\
\text { en p. } 100 \\
\text { à } 24 \mathrm{~h}\end{array}$} & \multirow{3}{*}{$\begin{array}{l}\text { Temps de } \\
\text { séjour en } \\
\text { cave chaude }\end{array}$} & \multirow{3}{*}{$\begin{array}{l}\text { Nombre de } \\
\text { fromages } \\
\text { butyriques (2) }\end{array}$} \\
\hline & \multicolumn{2}{|c|}{$6 \mathrm{~h}$} & \multicolumn{2}{|c|}{$24 \mathrm{~h}$} & & & \\
\hline & $\mathrm{C}$ & $\mathrm{T}$ & $\mathrm{C}$ & $\mathrm{T}$ & & & \\
\hline - & 5,66 & 5,56 & 5,18 & 5,17 & 60,6 & 53,2 & \\
\hline $\mathrm{Ct}$ & 5,66 & 5,50 & 5,21 & 5,22 & 61,4 & 48,0 & $5 \mathrm{~B}$ \\
\hline L. plantarum $3+343+\mathrm{Ct}$ & 5,58 & 5,54 & 5,16 & 5,20 & 61,8 & 53,0 & $3 \mathrm{~B}$ \\
\hline L. casei $\mathrm{K} 6+316+\mathrm{Ct}$ & 5,59 & 5,56 & 5,22 & 5,22 & 62,0 & 49,0 & $5 \mathrm{~B}$ \\
\hline L. helveticus $45+\mathrm{Ct}$ & 5,50 & 5,40 & 5,18 & 5,16 & 61,2 & 55,2 & $4 \mathrm{~B}$ \\
\hline Mélange cinq souches $+\mathrm{Ct}$ & 5,51 & 5,45 & 5,16 & 5,16 & 61,4 & 50,0 & $3 \mathrm{~B}$ \\
\hline
\end{tabular}

(1) Pour chaque groupe cinq fromages ont été fabriqués.

(2) Voir légende tableau 3. 


\section{DISCUSSION}

Les bactéries qui ont exercé une activité inhibitrice sur la croissance de $C$. tyrobutyricum appartiennent à des espèces dont certaines șouches ont déjà été signalées comme capables d'inhiber $C$. tyrobutyricum $[6,7,12,20,24]$ ou d'autres bactéries $[4,5,10,16,21,26,27$, $34,35]$. Par contre la souche NCDO 151 de $L$. casei mentionnée comme active contre $C$. tyrobutyricum [24] s'est avérée sans effet lorsqu'on l'a examinée au cours de la sélection des souches inhibitrices. Mais il est possible que la souche que nous avons utilisée ait été modifiée au cours de la conservation dans notre collection et ne corresponde plus à celle étudiée par ailleurs [24].

Quelle que soit la souche, l'inhibition s'exerçait sur la croissance végétative de $C$. tyrobutyricum et non sur la germination de la spore.

Contrairement à ce qui a été trouvé précédemment, aucune des souches étudiées dans ce travail n'agissait grâce à la formation d'eau oxygénée comme c'est le cas pour L. plantarum et d'autres lactobacilles vis-à-vis de $C$. tyrobutyricum $[6,7,35]$ ou d'autres espèces bactériennes $[3,10,27]$. La meilleure preuve est que la catalase ne supprimait pas l'effet inhibiteur des souches étudiées.

Les composés inhibiteurs n'étaient d'ailleurs pas dialysables ni même libérés dans le milieu de culture comme c'est le cas en particulier pour la souche NCDO 151 de $L$. casei qui inhibe $C$. tyrobutyricum [24]. Il ne s'agissait donc pas de métabolites comme par exemple l'acide acétique $[26,31]$ ou des antibiotiques comme la nisine $[13,15]$, la diplococcine [25] ou d'autres substances solubles non identifiées $[11,12,24,28,32]$ formées par différentes bactéries lactiques appartenant aux espèces étudiées ici ou à des espèces voisines.

Comme le montrent les résultats, l'inhibition ne se produisait qu'en présence de la fraction cellulaire des cultures, il pouvait donc s'agir de composés déjà présents dans les cellules mais qui ne seraient libérés qu'au cours du test d'inhibition en anaérobiose ou de composés qui ne se formeraient qu'au cours de ce test. Les lysats et les fractions solubles de cellules traitées aux ultrasons étaient inactifs, les composés inhibiteurs pourraient être liés aux débris cellulaires mais il n'était pas possible de conclure sur ce point car ces fractions contenaient encore des cellules viables. Toutefois si c'est le cas, les composés en cause n'étaient pas thermostables puisque les cellules chauffées étaient inactives. Comme le traitement au chloroforme supprimait également l'inhibition, celle-ci serait liée à la présence de cellules viables, la seconde hypothèse semble donc Ia plus vraisemblable. Il ne s'agit ni d'eau oxygénée, ni de l'acide 
lactique pour les raisons évoquées ci-dessus ou dans les résultats. Les composés inhibiteurs produits étaient capables de diffuser dans la gélose mais ne semblaient pas dialysables puisqu'une membrane intercalée entre les disques et le milieu ensemencé en $C$. tyrobutyricum supprimait l'inhibition. Comme il a été montré que certains streptocoques ne produisent une substance inhibitrice qu'en milieu gélosé [37], nous avons effectué des cultures des souches actives en présence de gélose et dans les mêmes conditions que le test de diffusion c'est-à-dire en anaérobiose. Les milieux de culture débarrassés des cellules étaient inactifs en diffusion. Toutefois cela n'est pas une preuve que des composés actifs solubles ne peuvent pas se produire dans ces conditions ; en effet un seul prélèvement a été effectué, or dans le cas des streptocoques du groupe A [37] la quantité d'inhibiteurs produits variait considérablement en fonction de l'âge de la culture en particulier en raison de son instabilité.

L'inhibition de $C$. tyrobutyricum pourrait être due également à la disparition d'un constituant du milieu indispensable à sa croissance, celui-ci étant consommé par les cellules des souches inhibitrices présentes dans les disques de papier, ce type d'antagonisme a déjà été signalé dans le cas des bactéries lactiques [10]. Toutefois cette hypothèse est peu vraisemblable pour les raisons suivantes. L'inhibition se produisait dans des milieux de composition et de richesse différentes, RCM, MRS et milieu au lactate, mais pas quand une membrane de dialyse était intercalée alors que celle-ci devrait permettre le passage des constituants du milieu tels que les vitamines. Enfin on comprend mal pourquoi ce phénomène ne se manifesterait qu'avec six des deux-cent-cinquante-deux souches examinées.

Des phénomènes d'inhibition bactérienne nécessitant la présence de cellules viables ont déjà été signalés sans qu'on puisse toujours déterminer la nature du mécanisme en cause $[4,18,19,26,34]$, dans certains cas les substances responsables ont d'ailleurs été isolées ultérieurement [37]. En ce qui concerne l'inhibition de $C$. tyrobutyricum par les souches que nous avons sélectionnées, il serait nécessaire d'entreprendre une étude plus approfondie souche par souche ou au moins espèce par espèce, car le mécanisme en cause n'est certainement pas le même pour toutes. Il faudrait en particulier revoir les possibilités de production d'inhibiteurs en milieu gélosé et dans les conditions d'anaérobiose de même que la possibilité de l'intervention d'enzymes, enzymes lytiques [17] ou lipases [33] libérant des acides gras susceptibles d'inhiber Clostridium tyrobutyricum.

En ce qui concerne l'utilisation de ces souches en vue d'empêcher le développement de $C$. tyrobutyricum et donc le gonflement butyrique dans les fromages, si les résultats de la première série d'essais étaient encourageants, les suivants et surtout les derniers étaient nettement moins bons. La principale différence qui a été 
observée entre les fromages de la première série et les autres concernait le $\mathrm{pH}$ et surtout son évolution au cours du pressage. Il est donc possible que le facteur qui ait joué, ait été l'acidification plus poussée plutôt qu'une variation dans l'inhibition propre aux souches utilisées, on sait en effet que c'est un facteur important dans l'apparition du gonflement butyrique.

Le fait que les souches inhibant $C$. tyrobutyricum en culture n'aient qu'un effet limité et non reproductible dans le fromage peut avoir plusieurs causes. D'abord il n'est pas sûr que ces souches se multiplient bien dans le fromage à part le cas de L. helveticus où on a pu le constater directement. Ensuite même si elles atteignent des nombres suffisants, il est possible que les facteurs inhibiteurs ne soient pas produits ou le soient mais en quantité insuffisante ou trop lentement pour inhiber complètement le développement de C. tyrobutyricum. Le fait que les fromages présentent une activité inhibitrice ne constitue pas une preuve suffisante, il a d'ailleurs déjà été constaté qu'il n'y avait pas de corrélation entre l'aptitude des fromages à inhiber $C$. tyrobutyricum déterminée par la méthode de diffusion et la présence de souches inhibitrices [12].

Les difficultés pour mettre en évidence les composés responsables de l'inhibition ayant montré que les conditions de production doivent être importants, il est possible que le fromage ne constitue pas un milieu convenable pour leur formation. Nous avons constaté également que l'inhibition ne se manifeste qu'en présence de cellules vivantes, or les bactéries inhibitrices ne se multiplient certainement pas d'une manière homogène dans les fromages et il est possible que leur répartition ne corresponde pas à celle de $C$. tyrobutyricum. Ceci est d'autant plus important que les composés inhibiteurs n'ont pas nécessairement une grande capacité de diffusion ni une stabilité suffisante dans les conditions qui règnent dans le fromage. Enfin on sait que dans le cas des antibiotiques comme la nisine, non seulement les souches productrices sont sensibles aux bactériophages mais sont aussi constituées de populations hétérogènes dans leur aptitude à produire ces composés et que certaines conditions favorisent le développement de populations qui n'en produisent pas [23]. $\mathrm{Ce}$ point devrait également être pris en considération dans une étude ultérieure sur la nature de l'inhibition. D'ailleurs si on connaissait mieux celle-ci, cela permettrait probablement d'utiliser les souches inhibitrices d'une manière moins empirique et peut-être avec plus d'efficacité. Toutefois s'il a été montré expérimentalement que l'emploi de souches antagonistes de $C$. tyrobutyricum peut empêcher le gonflement butyrique de se manifester dans les fromages $[8,20$, 23], il est important de remarquer que leur utilisation systématique dans les conditions de la pratique n'est pas sans poser de problèmes aussi bien en ce qui concerne les lactobacilles producteurs d'eau oxygénée que les souches productrices de nisine $[8,23$; Goudkov, 
communication personnelle]. C'est la raison pour laquelle ces méthodes n'ont pas connu de grand développement au stade de l'application, sauf dans le cas des fromages fondus et ceci malgré les nombreux travaux auxquels elles ont donné lieu. Ceci ne signifie pas que dans la pratique, la présence naturelle de ces bactéries inhibitrices (ainsi d'ailleurs que de celles qui sont stimulantes) et leur développement dans les fromages ne constitue pas un facteur important dans l'apparition ou non des défauts butyriques dans les fromages comme cela a été montré dans le cas du Cheddar [6]. C'est certainement une des raisons pour laquelle les fromages de divers types, ou de fabrications différentes à l'intérieur d'un même type, ne présentent pas la même sensibilité au gonflement butyrique.

\section{Remerciements}

Nous remercions le Laboratoire de la Chaire de Technologie de I'I.N.A. Paris-Grignon qui a bien voulu mettre sa collection de souches à notre disposition.

\section{Rés u m é}

Parmi une collection de deux-cent-cinquante-deux souches de diverses bactéries lactiques, six souches ont été reconnues comme capables d'inhiber $C$. tyrobutyricum par la méthode de diffusion en gélose, ce sont deux Lactobacillus casei, deux L. plantarum, un L. helveticus et un Streptococcus diacetylactis.

La nature exacte de l'inhibition n'a pu être déterminée, mais il ne s'agit ni de produit du métabolisme comme l'acide lactique, ni $d^{\prime} \mathrm{H}_{2} \mathrm{O}_{2}$, ni d'autres substances, antibiotiques ou non, libérées par les cellules en milieu liquide. L'inhibition ne se produit qu'en présence de cellules viables des souches inhibitrices.

Des essais d'utilisation de ces bactéries en fabrication fromagère ont montré qu'elles exercent également un effet inhibiteur sur le développement de C. tyrobutyricum dans des fromages d'Emmental. Toutefois cet effet est trop limité et insuffisamment reproductible pour qu'on puisse envisager actuellement de les employer pour contrôler le gonflement butyrique dans la pratique.

\section{S u m m a r y}

\section{LACTIC ACID BACTERIA HAVING INHIBITORY EFFECT \\ ON C. TYROBUTYRICUM \\ IN CULTURE AND IN CHEESE}

Out of two hundred fifty two strains of various lactic acid bacteria tested for inhibition of $C$. tyrobutyricum using the agar 
diffusion method, six strains were found to be capable of inhiting that species: two Lactobacillus casei, two L. plantarum, one L. helveticus and one Streptococcus diacetylactis.

The exact inhibition mechanism has not been demonstrated, yet metabolic products such as lactic acid are not involved nor $\mathrm{H}_{2} \mathrm{O}_{2}$ and other substances antibiotic or not released by the cells in liquid medium. Inhibition only occurs with viable cells from inhibitory strains.

These bacteria have been used in cheese production line and they have exhibited an inhibitory effect against the multiplication of C. tyrobutyricum in Emmental cheese. However this effect is too limited and insufficiently reproducible to consider its actual field application in controlling butyric blowing.

Reçu pour publication en février 1978.

\section{Bibliographie}

[1] Annibaldi (S.) (1963). - Effect of Streptococcus thermophilus on butyric acid fermentation in Grana cheese. Latte, 37, 54.

[2] Bergère (J. L.) (1969). - Germination de la spore de Clostridium tyrobutyricum. I. Action de différents composés sur la phase initiale. Ann. Inst. Pasteur, 117, 179.

[3] DahiYa (R. S.) and SPECK (M. L.) (1968), - Hydrogen peroxide formation by lactobacilli and its effect on Staphylococcus aureus. J. Dairy Sci., 51, 1568.

[4] Daly (C.), Sandine (W. E.) and Elliker (P. R.) (1972). - Interaction of food starter cultures and food-born pathogens: Streptococcus lactis versus food pathogens. J. Milk Food Technol., 35, 349.

[5] Gilliland (S. E.) and SPECK (M. L.) (1974). - Antagonism of lactic streptococci toward Staphylococcus aureus in associative milk cultures. Appl. Microbiol, 28, 1090.

[6] GoudKov (A. V.) and SHARPE (M. E.) (1966). - A preliminary investigation of the importance of Clostridia in the production of rancid flavour in Cheddar cheese. J. Dairy Res., 33, 139.

[7] Goudkov (A. V.) and Alekseeva (K. P.) (1970). - Interactions of lactobacilli with butyric acid bacteria causing cheese defect. Moloch. Prom., 31, 25.

[8] Goudkov (A. V.) and Dolidze (G. G.) (1974). - Prevention of butyric acid fermentation in cheese. Moloch. Prom., 7, 13.

[9] Goukt (P.) et BERgère (J. L.) (1973). - Ensilage et gonflement butyrique des fromages. Fourrages, 56, 89.

[10] Aines (W. C.) and Harmon (L. G.) (1973). - Effect of selected lactic acid bacteria on growth of Staphylococcus aureus and production of enterotoxin. Appl. Microbiol., 25, 436.

[11] Hamdan (I. Y.) and Mikolajcik (E. M.) (1974). - Acidolin: an antibiotic produced by Lactobacillus acidophilus. J. Antibiotics, 27, 631 . 
[12] Hirsch (A.), Mc. Clintock (M.) and Mocouot (G.) (1952). - Observations on the influence of inhibitory substances produced by the lactobacilli of Gruyere cheese on the development of anaerobic sporeformers. J. Dairy Res., 19, 179.

[13] Hirsch (A.), Grinsked (E.), Chapman (H. R.) and Mattick (A. T. R.) (1951). A note on the inhibition of an anaerobic sporeformer in Swiss-type cheese by a nisin producing Streptococcus, J. Dairy Res., 18, 205.

[14] Hunter (S. E.) and FrazieR (W. C.) (1961). - Gas production by associated swiss cheese bacteria. J. Dairy Sci., 44, 2176.

[15] HuRst (A.) (1973). - Interactions of food starter cultures and food borne pathogens: the antagonism between Streptococcus lactis and sporeforming microbes. J. Milk Food Technol., 35, 418.

[16] HuRst (A.) (1973). - Microbial antagonism in food. In microbial foodborne infections and intoxications. Hurst A. and de Man J.M., Ed., 33.

[17] Ishrwa (H.) and YoKoKura (T.) (1971). - A lytic enzyme (s) acting on lactobacilli. Jap. J. Microbiol., 15, 539.

[18] Kafel (S.) and Ayres (J. C.) (1969). - The antagonism of enterococci on other bacteria in canned hams. J. Appl. Bact., 32, 217.

[19] Kramer (J.) and Schallehn (G.) (1974). - Enterocinwirkung auf Clostridium perfringens und Clostridium septicum. Zentbl. Bakt. Parasitenk., $1 \mathrm{~A}$, 226,105 .

[20] KundRAT (W.) (1971). - Zur Bekämpfung der Spätblähung bei Hartkäsen auf biologischen Wegen. Alimenta, 4-5, 3 .

[21] LEE (E. C. T.) (1974). - Isolation and characterization of inhibitors for Pseudomonas fragi produced by Streptococcus diacetylactis. Dissertation Abst., 34, 3616 B.

[22] Lind (C. S.) (1956). - Studies on the growth of the Clostridia. XIV Int. Dairy, 3 (2), 276.

[23] LIPINSKA (E.) (1972), - Utilisation des streptocoques lactiques nisinogènes en fromagerie. Fed. Int. Lait., B. Doc. 14.

[24] Lodi (R.) et CARINI (S.) (1972). - Fenomeni di inibizione di Lactobacillus casei su Clostridium tyrobutyricum. XVI Congresso Nationale di Microbiologia Giardini, Ed., Pise, II, 329.

[25] OXFoRd (A. E.) (1944). - Diplococcin an anti-bacterial protein elaborated by certain milk streptococci. Biochem. J., 38, 178.

[26] Pinheiro (A. T. R.), Liska (B. J.) and Parmelee (C. E.) (1968). - Properties of substances inhibitory to Pseudomonas fragi oroduced by Streptococcus citrovorus and Streptococcus diacetylactis. J. Dairy Sci., 51, 183.

[27] PrICE (R. J.) and LeE (J. S.) (1970). - Inhibition of Pseudomonas species by hydrogen peroxide producing Lactobacilli. J. Milk and Food Technol., 33,13 .

[28] Reddy (G. V.) and Shahani (K. M.) (1971). - Isolation of an antibiotic from Lactobacillus bulgaricus. J. Dairy Sci., 54, 748.

[29] Roux (C.) et BERgère (J.L.) (1977). - Caractères taxonomiques de Clostridium tyrobutyricum. Ann. Microbiol., 128 A, 267.

[30] Richardson (G. H.) and Divatia (M. A.) (1973). - Lactic culture inocula required to inhibit staphylococci in sterile milk. J. Dairy Sci., 56, 706.

[31] SoRrells (K. M.) and SPECK (M. L.) (1970). - Inhibition of Salmonella gallinorum by culture filtrates of Leuconostoc citrovorum. J. Dairy Sci., 53, 239.

[32] Swartling (P.) and Lindgren (B.) (1960). - On the influence of certain bacteria on the growth of Clostridia. Report $n^{\circ}$ 62, Alnarp. 
[33] Unemoto (Y.), Umeda (H.) and Sato (Y.) (1968). - Studies on lipolysis of dairy lactic bacteria. II. On the lipolytic activities of cell free extracts of lactic acid bacteria. Agric. Biol. Chem., 32, 1311.

[34] Vedamuthu (E. R.), Hauser (B. A.), Henning (D. R.), Sandine (W. E.), Elliker (P. R.) and DAY (E. f.) (1966). - Compositive growth of Streptococcus diacetylactis in mixed strain lactic cultures and cheese. XVII Int. Dairy Cong., $D, 611$.

[35] Wheater (D. M.) Hirsch (A.) and Mattick (A. T. R.) (1952). - Possible identity of " lactobacillin " with hydrogen peroxide produced by lactobacilli. Nature, 170, 623 .

[36] Whittenbury (R.) (1964). - Hydrogen formation and catalase activity in the lactic acid bacteria. J. Gen. Microbiol., 35, 13.

[37] WolfF (L. F.) and Duncan (J. C.) (1974). - Studies on a bactericidal substance produced by group A streptococci. J. Gen. Microbiol., 81, 413. 